\title{
The HRV Multifractality Spectrum and not the Power Spectrum Is Altered in Paraplegic Individuals With Low-Level Lesion
}

\author{
Paolo Castiglioni ${ }^{1}$, Giampiero Merati ${ }^{1}$, Andrea Faini ${ }^{2}$ \\ ${ }^{1}$ IRCCS Fondazione Don Carlo Gnocchi, Milan, Italy \\ ${ }^{2}$ Istituto Auxologico Italiano, IRCCS, Milan, Italy
}

\begin{abstract}
The autonomic nervous system may contribute to the multifractal-multiscale dynamics of heart rate variability (HRV). Aim of this work is to evaluate multifractalmultiscale features of HRV complexity when the integrative autonomic regulation is altered. We recorded $R-R$ intervals for 10 minutes in 14 spinal cord injured (SCI) individuals with lesion below the 12th thoracic vertebra, which have intact autonomic cardiac innervations with partially altered autonomic integrative control, and in 34 matched able-bodied $(A B)$ controls. We calculated powers spectra by FFT and generalized Hurst coefficients, $\alpha(q, \tau)$, with scales $\tau$ between 6 and 120 seconds and $q$ between -4 and +4 , by multifractal multiscale detrended fluctuation analysis. At each scale we calculated $\alpha S D(\tau)$, standard deviation of $\alpha(q, \tau)$. $\alpha S D(\tau)$ is $\geq 0$ and tends to zero at scales where the series is monofractal. $A B$ and SCI groups were compared by Mann-Whitney test. While SCI and AB spectra were similar, $\alpha S D(\tau)$ was significantly lower at $\tau<7 \mathrm{~s}$ and at $\tau$ around $24 s$ in the SCI group. Thus, in low-level SCI individuals, the HRV multifractal-multiscale detrended fluctuation analysis reveals alterations in the autonomic integrative control, likely due to loss of multifractality at specific scales, that are completely undetected by traditional spectral analysis.
\end{abstract}

\section{Introduction}

Fractal analysis is becoming more and more popular for assessing the complex dynamics of cardiovascular time series [1]. In the field of heart-rate variability (HRV), the fractal characteristics are usually assessed by estimating the self-similarity scale coefficient $\alpha$ (strictly related to the Hurst's exponent) with the detrended fluctuation analysis method (DFA) [2]. Briefly, given a time series of $N$ samples $x_{i}$ with $i=1 \ldots N$ and mean $\mu$, the summation series $y_{i}$

$y_{i}=\sum_{j=1}^{i}\left(x_{j}-\mu\right)$

is split into $M$ blocks of $n$ consecutive samples.
DFA is based on the calculation of the variability function $F(n)$ :

$$
F(n)=\frac{1}{M} \sum_{k=1}^{M} \sqrt{\sigma_{n}^{2}(k)}
$$

with

$\sigma_{n}^{2}(k)=\frac{1}{n} \sum_{i=I_{k}}^{I_{k}+n-1}\left(y_{i}-p(i)\right)^{2}$

the variance of the $n$ consecutive samples in the $k$-th block, after detrending with the least-square fitting polynomial $p(i)$. The scale coefficient $\alpha$ is the slope of the regression line fitting $F(n)$ over $n$ in a log-log scale.

On one hand, some authors extended this single-scale approach estimating a continuous profile $\alpha(n)$, function of the scale $n$, by calculating the slope of the regression line over a running window centered on $n$ [3], [4]. In this way, DFA provides a multiscale representation of the fractal structure of HRV. On the other hand, DFA has been extended for estimating multifractality, defining the variability function as:

$$
\begin{cases}F_{q}(n)=\left(\frac{1}{M} \sum_{k=1}^{M}\left(\sigma_{n}^{2}(k)\right)^{q / 2}\right)^{1 / q} & \text { for } q \neq 0 \\ F_{q}(n)=e^{\frac{1}{2 M} \sum_{k=1}^{M} \ln \left(\sigma_{n}^{2}(k)\right)} & \text { for } q=0\end{cases}
$$

If $\alpha$, in this case estimated as slope of the regression line fitting $F_{q}(n)$ over $n$ in a log-log scale for each $q$ separately, depends on $q, \alpha(q)$, the series is multifractal [5].

More recently, the two approaches have been combined to provide a multifractal-multiscale representation of the scale coefficients, $\alpha(q, n)$, that allows quantifying the degree of multifractality separately at each scale [6]. In this way, DFA provides a more detailed and complete description of the complex dynamics of HRV.

Aim of this study is to apply the novel multifractalmultiscale approach for evaluating whether DFA can assess alterations of the integrative autonomic control of 
circulation by identifying loss of multifractality at specific scales. For this aim, we analyzed heart rate recordings in spinal-cord injured $(\mathrm{SCI})$ individuals with a low spinal lesion. These subjects have intact cardiac autonomic innervations but suffer from an impaired sympathetic control of the lower limbs and vascular districts, therefore representing a model of a partially impaired integrative autonomic control. We may expect that an impaired integrative control is associated with a loss of cardiovascular complexity.

\section{Methods}

We recorded R-R intervals (RRI) for 10 minutes in 14 spinal cord injured (SCI) individuals with lesion below the 12th thoracic vertebra and in 34 matched able-bodied (AB) controls. All participants were in sitting position at rest. RRI beat-by-beat series were interpolated evenly at 5 $\mathrm{Hz}$ before spectral analysis. The Welch periodogram was estimated by FFT with $90 \%$ overlapped Hann windows of $180 \mathrm{~s}$ length, and smoothed with a broadband procedure [7]. Power spectra were integrated over the very-low frequency (VLF, 0.005-0.04 Hz), the low-frequency (LF, $0.04-0.15 \mathrm{~Hz}$ ) and the high-frequency (HF, 0.15-0.40 $\mathrm{Hz}$ ) bands. Further details on spectral analysis can be found in [8].

The multifractal-multiscale DFA was performed on the beat-by-beat RRI series. To minimize the estimation variability of $F_{q}(n)$, the equation 1 was calculated splitting the series into maximally overlapped blocks of size $n$, as described in [9]. The multifractal variability function, $F_{q}(n)$, was estimated for a set of scales, $n_{k}$, with $k$ between 1 and $K$, equispaced over the logarithmic scale. Called $N$ the length of the time series, $K$ was set equal to $N / 4$. We considered 9 values of the $q$ parameter, equispaced between -4 and +4 . Local scale exponents $\alpha_{B}\left(q, n_{k}\right)$ were calculated at scales $n_{k}$, with $k$ between 2 and $K-1$, as the derivative of $\log F_{q}(n)$ vs. $\log n$ :

$\alpha_{B}\left(q, n_{k}\right)=\frac{\log F_{q}\left(n_{k+1}\right)-\log F_{q}\left(n_{k-1}\right)}{\log n_{k+1}-\log n_{k-1}}$

To move from the beat-domain into the time-domain, we applied the transformation:

$\alpha\left(q, \tau_{k}\right)=\alpha_{B}\left(q, n_{k}\right)$ with $\tau_{k}=n_{k} \times 60 / H R_{m}$

where $H R_{m}$ is the mean heart rate, in bpm. The multifractal-multiscale surface of DFA coefficients, $\alpha(q, \tau)$, was obtained linearly interpolating $\alpha\left(q, \tau_{k}\right)$ over the time scale between $5 \mathrm{~s}$ and $128 \mathrm{~s}$.

Furthermore, we calculated a concise index of the degree of multifractality at each scale separately. For any given $\tau^{\prime}$ scale, we calculated $\alpha_{\mathrm{SD}}\left(\tau^{\prime}\right)$ defined as the standard deviation of the nine $\alpha\left(q, \tau^{\prime}\right)$ values estimated for each of the nine parameters $q$ we considered. The $\alpha_{\mathrm{SD}}(\tau)$ index is expected to be substantially greater than zero at scales were the time series is multifractal, and to tend to zero at scales with monofractal dynamics.

\section{Results}

The two groups were very well matched in terms of age (AB: $39.3 \pm 12.1$; SCI: $40.9 \pm 10.0 \mathrm{yrs}$ ), BMI (AB: $24.7 \pm 2.6$; SCI: $25.5 \pm 4.8 \mathrm{~kg} / \mathrm{m}^{2}$ ) and gender ratio (AB: 5/29; SCI: 2/12 Females/Males).

The average power spectra calculated over the $A B$ and SCI groups were practically superimposable. In particular, the difference between $\mathrm{AB}$ and SCI groups for each spectral line was far from the statistical significance over the whole frequency axis (see figure 1).

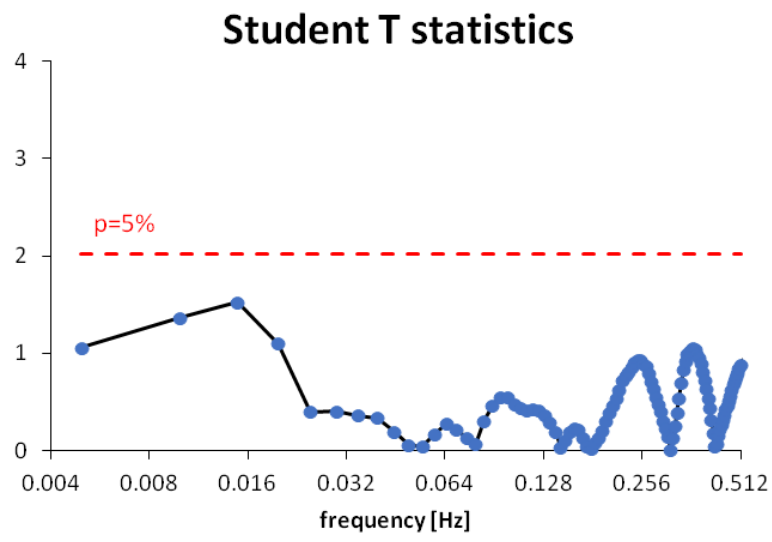

Figure 1. Statistical significance of the difference in RRI power spectra between $\mathrm{AB}$ and SCI groups by Student $\mathrm{t}$ test after log transformation. The horizontal dashed line is the statistical threshold at $5 \%$ significance: since the threshold is never crossed, the $\mathrm{AB}$ and SCI power spectra do not differ significantly at any frequency.

The power spectra in the VLF, LF and HF frequency bands are reported in table 1.

Table 1. Power spectra of RRI in AB and SCI groups, as mean $\pm \mathrm{SD}$, with $\mathrm{p}$ the statistical significance of the difference between groups.

\begin{tabular}{llll}
\hline $\begin{array}{l}\text { Spectral } \\
\text { Parameter }\end{array}$ & $\mathrm{AB}$ & $\mathrm{SCI}$ & $\mathrm{p}$ \\
\hline VLF & $2.8 \pm 0.4$ & $2.9 \pm 0.3$ & 0.34 \\
LF & $2.8 \pm 0.5$ & $2.8 \pm 0.4$ & 0.84 \\
HF & $2.3 \pm 0.5$ & $2.3 \pm 0.5$ & 0.83 \\
LF/HF & $0.6 \pm .03$ & $0.6 \pm .02$ & 0.72 \\
\hline
\end{tabular}

VLF, LF and HF powers as $\log _{10} \mathrm{~ms}^{2} / \mathrm{Hz}$; p after MannWhitney test

The LF and HF powers and their ratio were identical in the $\mathrm{AB}$ and SCI groups. The VLF power was slightly greater in the SCI group, the difference however being far from the statistical significance. 


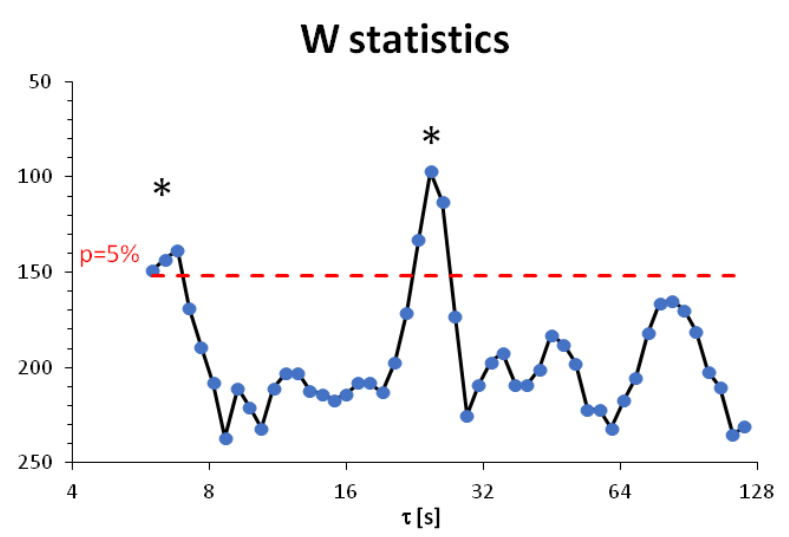

Figure 2. Mann Whitney $\mathrm{W}$ statistics testing the difference between $\mathrm{AB}$ and SCI groups in $\alpha_{\mathrm{SD}}(\tau)$; the horizontal dashed line is the threshold at $5 \%$ statistical significance. $\mathrm{W}$ values above the threshold are associated with significant differences and are marked by asterisks.

By contrast, differences between groups were found in $\alpha_{\mathrm{SD}}(\tau)$, index representing multifractality at each scale separately. Figure 2 represents the $\mathrm{W}$ statistics resulting from the Mann Whitney test comparing $\mathrm{AB}$ and SCI group. The statistics is represented as function of the scale $\tau$. The figure points out significant differences at scales shorter than $7 \mathrm{~s}$ and at scales around $24 \mathrm{~s}$.

At both these scales $\alpha_{\mathrm{SD}}(\tau)$ is significantly lower in the SCI group. In particular, at $\tau \leq 7 \mathrm{~S} \alpha_{\mathrm{SD}}(\tau)$ is $3.77 \pm 0.95$ in the AB group, $2.30 \pm 0.40$ in the SCI group (values as median \pm standard error of the median computed using bootstrap technique); at $23 \mathrm{~s} \leq \tau \leq 26 \mathrm{~s}, \alpha_{\mathrm{SD}}(\tau)$ is $1.80 \pm 0.19$ in the AB group, $0.99 \pm 0.20$ in the SCI group.

\section{Discussion}

The SCI group represents a human model of intact autonomic cardiac innervation with impaired autonomic integrative control of circulation due to an altered sympathetic control of the lower vascular districts. This human model is characterized by preserved spectral components, as testified by the comparison of their power spectra with those of a group of controls with the same age, body mass and sex composition.

By contrast, clear alterations appear in the HRV dynamics when complexity features are assessed by means of the multifractal-multiscale approach to DFA. The comparison with the control group points out that the alterations consist in a loss of HRV multifractality, concentrated at specific scales.

Therefore, the multifractal-multiscale DFA of cardiovascular time series appears to be a promising tool for detecting alterations in the autonomic integrative control of circulation. Such alterations may go completely undetected if evaluated by traditional spectral methods.

\section{References}

[1] P. Castiglioni, M. Di Rienzo, and A. Faini. Self-similarity and detrended fluctuation analysis of cardiovascular signals. In Complexity and Nonlinearity in Cardiovascular Signals, R. Barbieri, E. P. Scilingo, and G. Valenza, Eds. Cham: Springer International Publishing, 2017, pp. 197232.

[2] C. K. Peng, S. Havlin, H. E. Stanley, and A. L. Goldberger. Quantification of scaling exponents and crossover phenomena in nonstationary heartbeat time series. Chaos., vol. 5, no. 1054-1500, pp. 82-87, 1995.

[3] J. Xia, P. Shang, and J. Wang. Estimation of local scale exponents for heartbeat time series based on DFA. Nonlinear Dynamics, vol. 74, no. 4, pp. 1183-1190, 2013.

[4] P. Castiglioni, G. Parati, A. Civijian, L. Quintin, and M. Di Rienzo. Local scale exponents of blood pressure and heart rate variability by detrended fluctuation analysis: effects of posture, exercise, and aging. IEEE Trans Biomed Eng, vol. 56, no. 1558-2531 (Electronic), pp. 675-684, Mar. 2009.

[5] J. W. Kantelhardt, S. A. Zschiegner, E. Koscielny-Bunde, S. Havlin, A. Bunde, and H. E. Stanley. Multifractal detrended fluctuation analysis of nonstationary time series. Physica A: Statistical Mechanics and its Applications, vol. 316, no. 1-4, pp. 87-114, Dec. 2002.

[6] J. Gieraltowski, J. J. Zebrowski, and R. Baranowski. Multiscale multifractal analysis of heart rate variability recordings with a large number of occurrences of arrhythmia. Phys.Rev.E Stat.Nonlin.Soft.Matter Phys., vol. 85, no. 1550-2376 (Electronic), p. 021915, Feb. 2012.

[7] M. Di Rienzo, P. Castiglioni, G. Parati, G. Mancia, and A. Pedotti. Effects of sino-aortic denervation on spectral characteristics of blood pressure and pulse interval variability: a wide-band approach. Med Biol Eng Comput., vol. 34, no. 0140-0118 (Print), pp. 133-141, Mar. 1996.

[8] P. Castiglioni and G. Merati. Fractal analysis of heart rate variability reveals alterations of the integrative autonomic control of circulation in paraplegic individuals. Physiological Measurement, vol. 38, no. 5, pp. 774-786, 2017.

[9] P. Castiglioni, D. Lazzeroni, P. Coruzzi, and A. Faini. Multifractal-multiscale analysis of cardiovascular signals: a dfa-based characterization of blood pressure and heartrate complexity by gender. Complexity, vol. 2018, pp. $1-$ $14,2018$.

Address for correspondence.

Paolo Castiglioni.

IRCCS Fondazione Don C. Gnocchi

Via Capecelatro 66, I 20148, Milano, Italy.

E-mail: pcastiglioni@dongnocchi.it 